\title{
Recherches sur la toxicité et la valeur trypanopréventive du moranylate d'éthidium
}

\author{
III. Modification de la toxicité et pouvoir préventif
}

por J. BALIS.

Le moranylate d'éthidium a déjà fait l'objet de plusieurs publications qui ont mis en évidence ses remarquables propriétés préventives et curatives.

Son emploi a été très limité en raison des réactions très importantes qu'it provoque; it était donc intéressant d'étudier différents moyens susceptibles de diminuer ces réactions tout en conservant autant que possible la totalité des qualités préventives.

Après un exposé succinct des propriétés physico-chimiques du complexe, nous avons étudié les réactions tant locales que générales provoquées par l'administration de moranylate d'éthidium en injection sous-cutanée, associé ou non à différents adjuvants, sous forme d'implants ou en injection intraveineuse.

Les animaux traités ont ensuite été placés au Nord-Cameroun dans notre station de RIGGIL qui est une zone d'infestation moyenne.

\section{PROPRIÉTÉS PHYSICO-CHIMIQUES}

Nous nous bornerons à dire que le moranylate d'éthidium se présente sous l'aspec: d'une poudre rouge et résulte de la combinaison de :

Moranyl acide 40 p. 100.

Bromure d'éthidium (hydroxyde) 60 p. 100.

Il est insoluble dans la plupar: des solvants organiques sauf les alcools méthylique et amylique. Il est très peu soluble dans l'eau $(0,0007 \mathrm{p}$. $100)$, un peu plus dans le sérum physiologique $(0,0017)$ et dans le sérum de bœuf $(0,0045 p, 100)$.

La plus grande solubilité dans l'eau physiologique peut être expliquée en interprétcn: l'observation suivante :

Reçu pour publication : février 1961.

Rev. Elev. Méd. vét. Pays trop., 1961, 14, no 2.
On effectue les mélanges :

a) Moranylate d'éthidium $+\mathrm{NaCl}+$ eau distillée (à l'ébullition),

b) Moranylate d'éthidium $+\mathrm{NaCl}+$ eau distillée (à froid),

c) Moranylate d'éthidium + eau distillée (ì froid),

d) Ethidium + equ distillée.

On dépose une goutte de chaque mélange sur un papier buvard ; après dessiccation on observe en lumière de WOOD que la fluorescence est la même en (o) et (d). En (b) elle est plus faible et en (c) elle est très faible.

Il y a donc vraisemblablement un début de dissociation en eau distillée ef ce phénomène s'accentue sous l'influence du chlorure de sodium et de la chaleur avec formation dans ce dernier cas de chlorure d'éthidium et de moranylate de sodium. Il est probable que l'effet préventif est dồ la libération lente d'éthidium.

Dans le sérum sanguin, outre le dissociation, interviennent également des phénomènes d'adsorption.

Le moranylate d'éthidium n'est utilisable qu'en suspension et le taux de 10 p. 100 s'est avéré le plius pratique.

\section{MODIFICATIONS DE LA TOXICITÉ}

\section{1. - Toxicité locale}

a) Utilisation de gel d'alumine comme adiuvant.

La suspension de moranylate d'éthidium étant beaucoup plus stable en présence de gel d'alumine, nous l'avons utilisée de la façon suivante sur le bouvillon no 25 : 


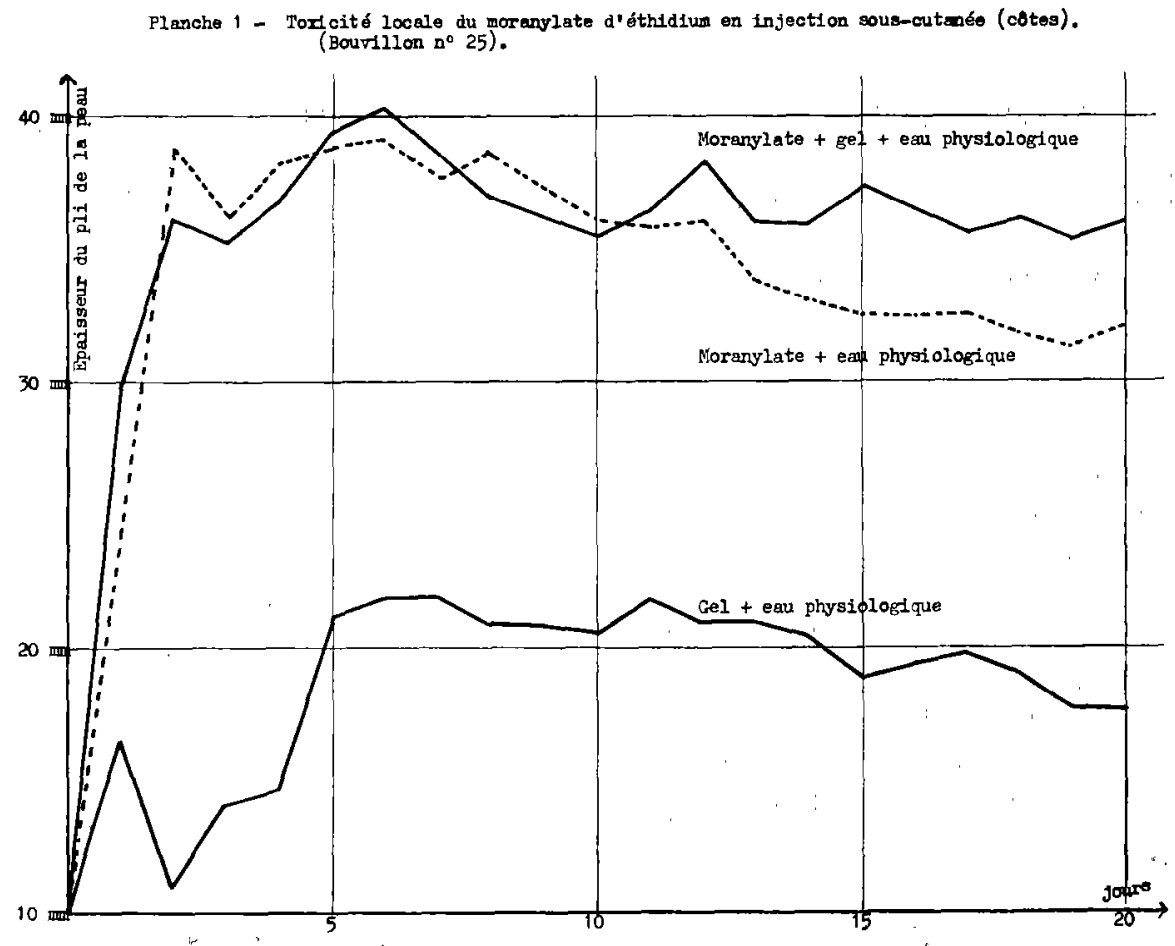

Planche 2 - Toxfeité locale sy moranylate d'éthidium en injection sous-cutanée (fanon).

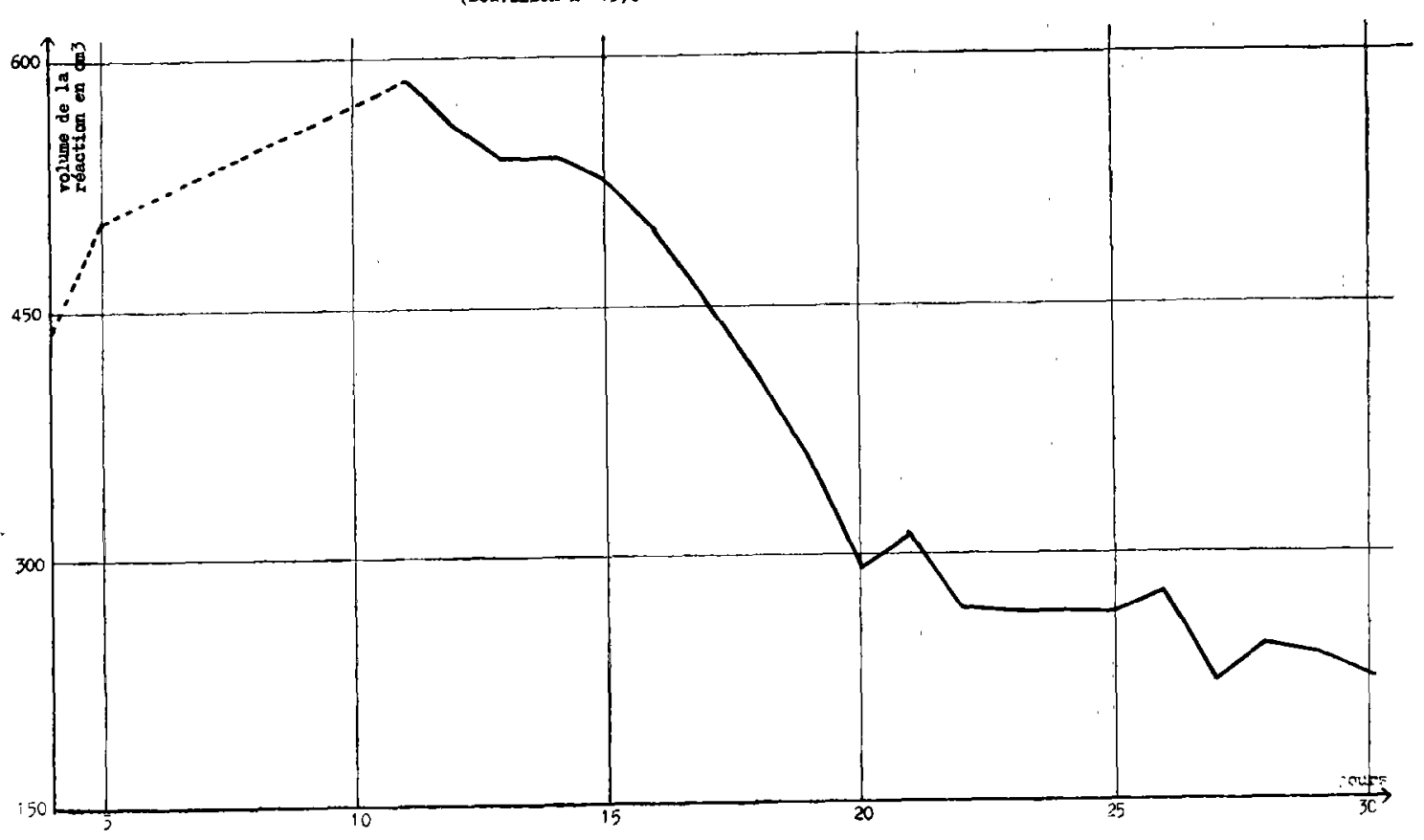


De chaque côté du thorax on a rasé 3 rangées de 5 plages de $4 \mathrm{~cm}$ de largeur environ et distantes l'une de l'autre de $10 \mathrm{~cm}$ environ, puis les préparations suivantes ont été réparties chacune en 10 injections de $0,5 \mathrm{ml}$.

$3 \mathrm{~g}$ de gel + QS eau physiologique pour $5,5 \mathrm{~g}$,

$3 \mathrm{~g}$ de gel $+250 \mathrm{mg}$ de moranylate d'éthidium + QS eau physiologique pour $5,5 \mathrm{~g}$.

$250 \mathrm{mg}$ de moranylate d'éthidium + QS eau physiologique pour $5.5 \mathrm{~g}$.

L'importance de la réaction a été mesurée chaque jour au pied à coulisse en faisant faire un pli à la peau. Les valeurs trouvées en millimètres ont servi à l'établissement de 3 graphiques (planche I). En ordonnée se trouvent les valeurs moyennes correspondant à $0,5 \mathrm{ml}$, en abscisse, le temps en jours.

b) Utilisation de la lanoline comme excipient avec ou sans cortisone.

Bouvillon no 46 :

Moranylate d'éthidium en suspension lanolinée à 30 p. 100 ;

Moranylate d'éthidium en suspension lanolinée à 30 p. $100+$ cortisone à 6 p. 100 ;

Moranylate d'éthidium en suspension aqueuse à 10 p. 100.

Les injections ont été faites sur chaque face thoracique de la façon suivante :

côté droit : 2 rangées de 2 plages.

rangée supérieure:

$0,25 \mathrm{ml}$ de suspension à $30 \mathrm{p} .100$ soit $75 \mathrm{mg}$ de moranylate d'éthidium.

$0,50 \mathrm{ml}$ de suspension à 30 p. 100 soit $150 \mathrm{mg}$ de moranylate d'éthidium.

rangée inféricure :

$0,25 \mathrm{ml}$ de suspension à 30 p. $100 \div 6$ p. 100 de cortisone,

$0,50 \mathrm{ml}$ de suspension à 30 p. $100+6$ p. 100 de cortisone.

côté gouche : une seule rangée de 2 plages :

$75 \mathrm{mg}$ de moranylate d'éthidium en suspension aqueuse à 10 p. 100 ,

$150 \mathrm{mg}$ de moranylate d'éthidium en suspension aqueuse à 10 p. 100 .

c) Utilisation d'un excipient huileux ovec ou sans cortisone.

\section{Bouvillon $n^{\circ} 61$ :}

côté droit : 2 rangées de 3 injections :

rongée supérieure :

Moranylate d'éthidium en suspension huileuse $25 \mathrm{mg}, 50 \mathrm{mg}$ et $100 \mathrm{mg}$.

rongée inférieure :

Moranylate d'éthidium en suspension huileuse aux mêmes doses + cortisone.

cófé gouche: 3 injections de moranylate d'éthidium en suspension aqueuse aux mêmes doses.

Ces deux bouvillons nous ont permis de constater qu'un excipient huileux ou lanoliné réduit la réaction et que, par contre, la cortisone, a tendance à l'augmenter.

La traduction de l'ensemble des résultats en pourcentage nous donne les valeurs suivantes par rapport au moranylate sans adjuvant :

Suspension lanolinée ........... 79 p. 100

Suspension lanolinée + cortisone. . 107 p. 100

Suspension huileuse ............ 84 p. 100

Suspension huileuse + cortisone... 94 p. 100

Bouvillon no 15. :

Il a subi une injection de moranylate d'éthidium en suspension huileuse dans le fanon, à la dose de $5 \mathrm{mg} / \mathrm{kg}$. La réaction n'a pas dépassé le volume de $600 \mathrm{~cm}^{3}$; un graphique en indique l'allure (planche 2).

Botivilion no 80 . :

Même traitement et réaction du même type.

d) Moranylate d'éthidium sous forme d'implants flus ou moins délitables avec ou sans cortisone :

Bouvillons nos 23 ef 19 . :

Nous avons utilisé des implants de $100 \mathrm{mg}$ environ chacun, introduits sous la peau selon un protocole analogue à ceux déjà employés pour les nos 25,46 et 61 . Comme toujours, les résultats ont été relevés chaque jour au pied à coulisse.

côté droit : de droite à gauche :

Un implant non délitable $=$ nd

Un implant peu délitable $=p d$

Un implant délitable $=d$ 
côté gauche :

Un implant non délitable + cortisone $=$ nd $+\mathrm{c}$

Un implant peu délitable + cortisone $=$ pd $+c$

Un implant délitable + cortisone $=d+c$

Parallèlement 6 bouvillons ont été traités chacun avec 1 implant de $500 \mathrm{mg}$ introduit vers le bas, dans le fanon, à l'aide d'un trocard plat spécial. Ce sont les numéros suivants :

$$
\begin{array}{ll}
n^{\circ} 24 \text { nd } & n \circ 5 n d+c \\
n^{\circ} 79 p d & n \circ 8 p d+c \\
n \circ 12 p & n \circ 6 d+c
\end{array}
$$

L'épaisseur du fanon a été mesurée chaque jour au pied à coulisse pendant 42 jours. La plaie provoquée par le trocard s'est rapidement cicatrisée et en aucun cas nous n'avons constaté de fistules. Deux graphiques indiquent l'allure des réactions avec et sans cortisone (planche 3 ).
TABLEAU Nn 1

\begin{tabular}{|c|c|c|c|}
\hline $\begin{array}{c}\text { No des bou- } \\
\text { villons }\end{array}$ & Poids & $\begin{array}{c}\text { Total des } \\
\text { implants }\end{array}$ & $\begin{array}{c}\text { Dose en } \\
\mathrm{mg} / \mathrm{kg}\end{array}$ \\
\cline { 1 - 2 } 24 & 151 & 6 & 20 \\
\hdashline & 157 & 6 & 20 \\
6 & 134 & 4 & 15 \\
12 & 179 & 5 & 14 \\
5 & 212 & 6 & 14 \\
79 & 147 & 3 & 10 \\
8 & 145 & 3 & 10 \\
\hline
\end{tabular}

Nous avons pu, tirer les conclusions suivantes de l'ensemble de ces expérimentations :

1. - Le moranylate d'éthidium est une substance très irritante, provoquant, en suspension à 10 p. 100 et par voie sous-cutanée, avec ou sans gel d'alumine, une forte réaction pouvant aboutir à des escarres à partir de $50 \mathrm{mg} / \mathrm{kg}$; cependant certains animaux peuvent supporter $150 \mathrm{mg}$ et ne manifester qu'une grosse induration.

Planche 3 - Toxicité locale du moranylate d'ćthidium introduit sous la peau du fanon sous fortie d'implants.

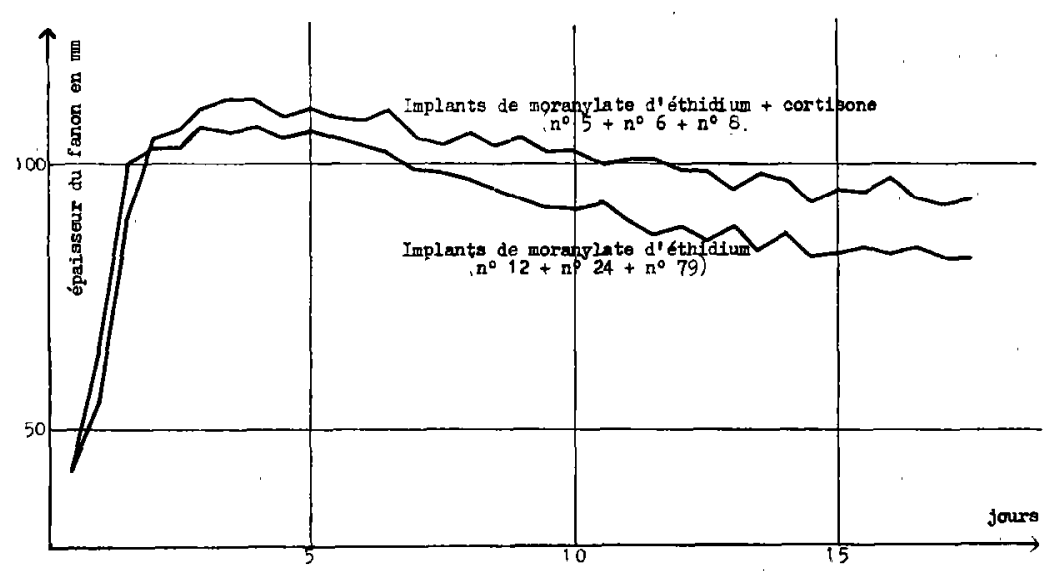

Sur l'un des graphiques nous avons, en ordonnée, la somme des valeurs trouvées pour 3 bouvillons sans cortisone ; sur l'autre, la somme des valeurs avec cortisone. En abscisse, est porté le temps, en jours. Les réactions étant acceptables, nous avons repris les numéros précédents avec, en plus, le $n^{0} 25$ pour un traitement avec implants délitables à des doses variant de 20 à $10 \mathrm{mg} / \mathrm{kg}$. Tous les renseignements sont consignés dans le tableau no 1.
2. - La réaction mesurée en volume est direc tement proportionnelle a la dose, tout au moins quand celle-ci est faible. Ceci n'est valable que si les quantités variables sont injectées sur le même animal car chacun réagit d'une façon différente.

3. - Le moranylate d'éthidium en suspension huileuse est nettement mieux toléré qu'en suspension aqueuse et il est possible d'injecter une dose de $5 \mathrm{mg} / \mathrm{kg}$ au niveau du fanon sans 
réaction excessive. Cette voie d'introduction sous-cutanée semble la meilleure.

4. - La cortisone augmente nettement ia réaction qui devient tout à fait comparable à celle donnée par la suspension aqueuse de moranylate d'éthidium.

5. - La suspension Ianolinée provoque des réactions analogues a celles obtenues avec la suspension huileuse, mais, étant très difficile à injecter avec un matériel courant, elle ne présente aucun intérêt pratique.

6. - Les implants de moranylate d'éthidium, au niveau du fanon, avec ou sans cortisone sont parfaitement supportés par les animaux qui n'ont jamais présenté de réactions importantes. Sur les 7 animaux traités de la sorte à des doses allant jusqu'à $20 \mathrm{mg} / \mathrm{kg}$, un seul (15 mg/ $/ \mathrm{kg}$ ) a présenté une fistulisation au bout de 5 mois.

Comme précédemment, la cortisone augmente la valeur des réactions.

7. - Les 3 formes d'implants utilisés : non délitables, peu délitables et délitables provoquent les mêmes réactions.

\section{2. - Toxicité générale}

a) Voie sous-cutanée :

Elle est nulle pour les implants et semble faible pour des doses inférieures à $5 \mathrm{mg} / \mathrm{kg}$ en suspension huileuse ou aqueuse; elle se manifeste alors par une action légèrement défavorable sur la croissance des animaux pendant les premiers mois. Aucune modification de la courbe thermique n'a été constatée.

\section{b) Voie intra-veineuse :}

L'un de nos confrères a montré que le moranylate d'éthidium présente par voie intra-veineuse une toxicité certaine, mais le caractère irrégulier de cette dernière laisse penser qu'il s'agisrait peut-être de particules s'embolisant dans les capillaires ef provoquant des petits foyers d'inflammation capables de causer des troubles graves au niveau du foie, du poumon et du cerveau.

C'est pourquoi nous avons repris l'expérience en partant du fait qu'un mélange de solutions de moranyl et d'éthidium en proportions convenables donne immédiatement un précipité de complexe. Nous avons injecté, par voie intraveineuse, les deux composants l'un après l'autre afin de provoquer la formation du précipité directement dans le sang et donc à l'état très divisé.

Le tableau $n^{\circ} 2$ résume l'opération:

Ont été traités aux dates suivantes :

le $21 / 3 / 60,3$ animaux à $5 \mathrm{mg} / \mathrm{kg}$ (moranyl + éthidium séparément) ;

le $7,5,60,3$ animaux à $10 \mathrm{mg} / \mathrm{kg}$ (morany! + éthidium séparément) ;

le 19:5,60, 3 animaux avec la dose d'éthidium correspondant à $5 \mathrm{mg} / \mathrm{kg}$ de complexe

le 7/5/60, 3 animaux avec la dose d'éthidium correspondant à $10 \mathrm{mg} / \mathrm{kg}$ de complexe.

Tous ces animaux ont été suivis ainsi que 3 témoins pendant plusieurs mois (frottis, gouttes épaisses, poids).

Nous n'avons eu que deux morts: I'un ( $n^{\circ} 13$ ) qui avait reçu une dose toxique d'éthidium $(0,6 \mathrm{mg} / \mathrm{kg})$, l'autre (no 2) traité à $10 \mathrm{mg} / \mathrm{kg}$ de complexe.

Une série de courbes (planche no 4), traduisant la variation de poids des animaux traités par rapport aux témoins, fait ressortir une chute pondérable dans le mois qui suit le traitement.

Bien que les différences constatées ne soient pas très importantes, il semble que l'injection d'éthidium seul soit plus toxique que lorsqu'on l'associe au moranyl.

Dès le mois de juillet, c'est-à-dire à la suite d'une alimentation plus abondante, tous les animaux ont repris normalement du poids.

La toxicité générale est donc presque acceptable quand on injecte le moranyl et l'éthidium l'un à la suite de l'autre. Malheureusement, nous verrons plus loin que, du point de vue trypanopréventif, le procédé ne donne aucun résultat.

\section{POUVOIR CURATIF}

Deux bouvillons étaient trypanosomés avant l'expérimentation: l'un a été traité avec une suspension huileuse, l'autre par implants et dans les deux cas les parasites ont régulièrement disparu en 24 heures.

\section{POUVOIR PRÉVENTIF}

Pour étudier la valeur trypanopréventive $\mathrm{du}$ moranylate d'éthidium dans les conditions natu- 
TABLDAU II - Protocole d'injeotion intraveineuse d'Ethidium et de Moranyl.

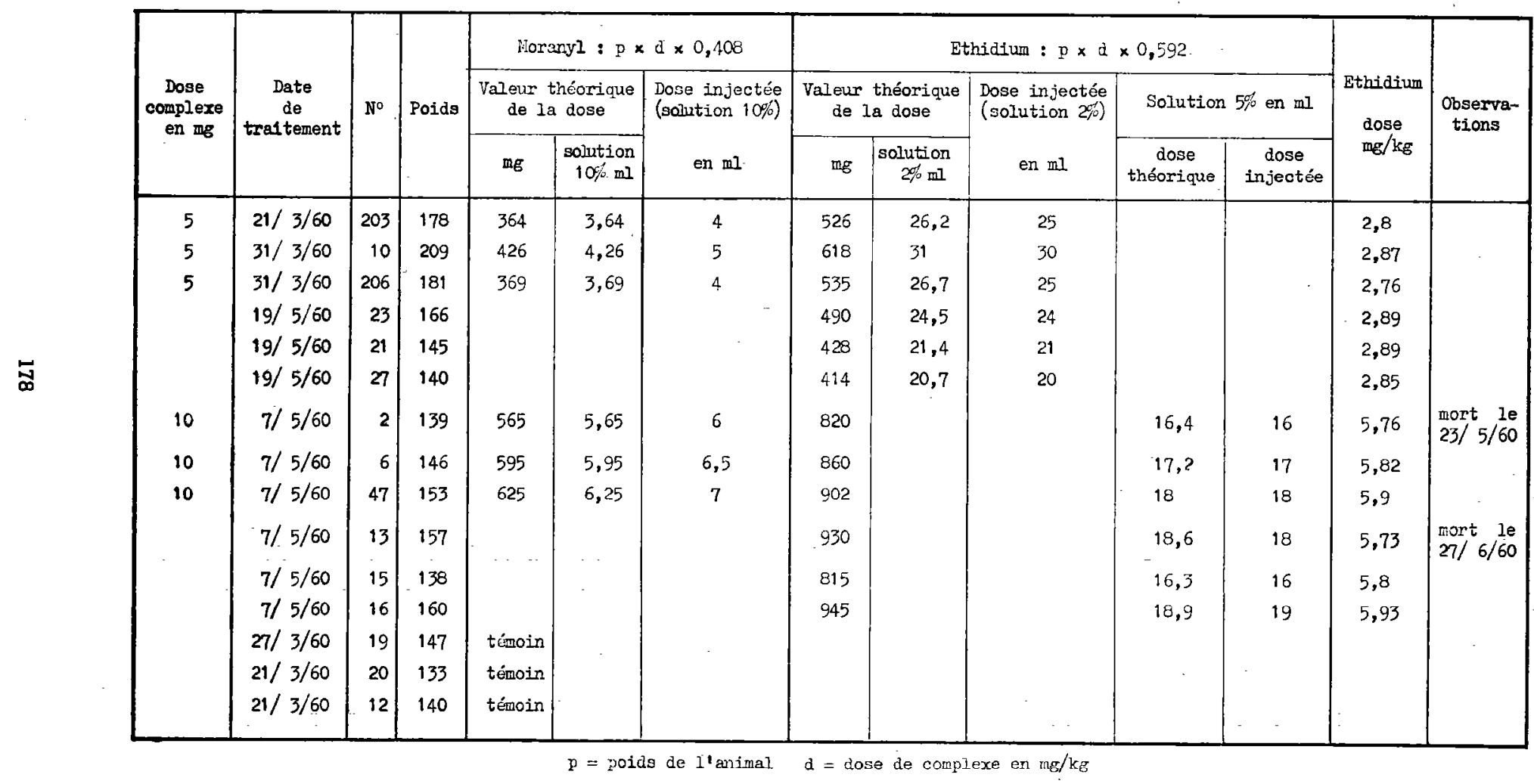


relles d'infestation, tous les animaux de l'expérimentation, qu'ils aient reçu le produit sous forme de suspension aqueuse ou huileuse ou d'implant, ont été transportés dans le NordCameroun à la station de Riggil. Là l'infestation trypanosomienne est celle que l'on trouve en brousse le long des cours d'eau.

Les suspensions huileuse et aqueuse donnent une trypanoprévention comparable. Par contre, cefte dernière est beaucoup plus nuancée pour ce qui est des implants: On constate en effet que la prévention absolve, c'est-à-dire le temps pendant lequel on ne trouve pas de trypanosomes dans le sang, est indépendante de la dose ; c'est ainsi qu'un bouvillon traité à $10 \mathrm{mg} / \mathrm{kg}$ est resté indemne pendant 16 mois alors qu'à l'opposé, un autre traité à $16 \mathrm{mg} / \mathrm{kg}$ n'a présenté qu'une prévention absolve de 3 mois. Mais alors que les témoins infestés font une trypanosomiase clas- sique aboutissant généralement à la mort en l'absence de traitement, tous les animaux ayant reçu des implants se conduisent comme des trypanotolérants; l'apparition de trypanosomes dans leur sang est toujours rare et très fugace.

Ceci contraste avec l'observation suivante :

Nous avons traité trois témoins trypanosomés par injections intra-veineuses de moranyl suivi d'éthidium à des doses (supposées) de complexe variant de 2 à $4 \mathrm{mg} / \mathrm{kg}$. La prévention a été dans tous les cas comprise entre deux et trois mois ; or certains de nos témoins mettent parfois plus de deux mois à s'infester. Il est donc vraisemblable qu'il n'y a pas combinaison dans le sang entre le moranyl et l'éthidium, ou encore, en admettant que le complexe se forme réellement, il est suffisamment divisé pour permettre son élimination rapide ef ne peut donc pas exercer un effet préventif de longue durée.

Planche 4 - Variation du poids des animaux traités avec le moranylate d'éthidium.

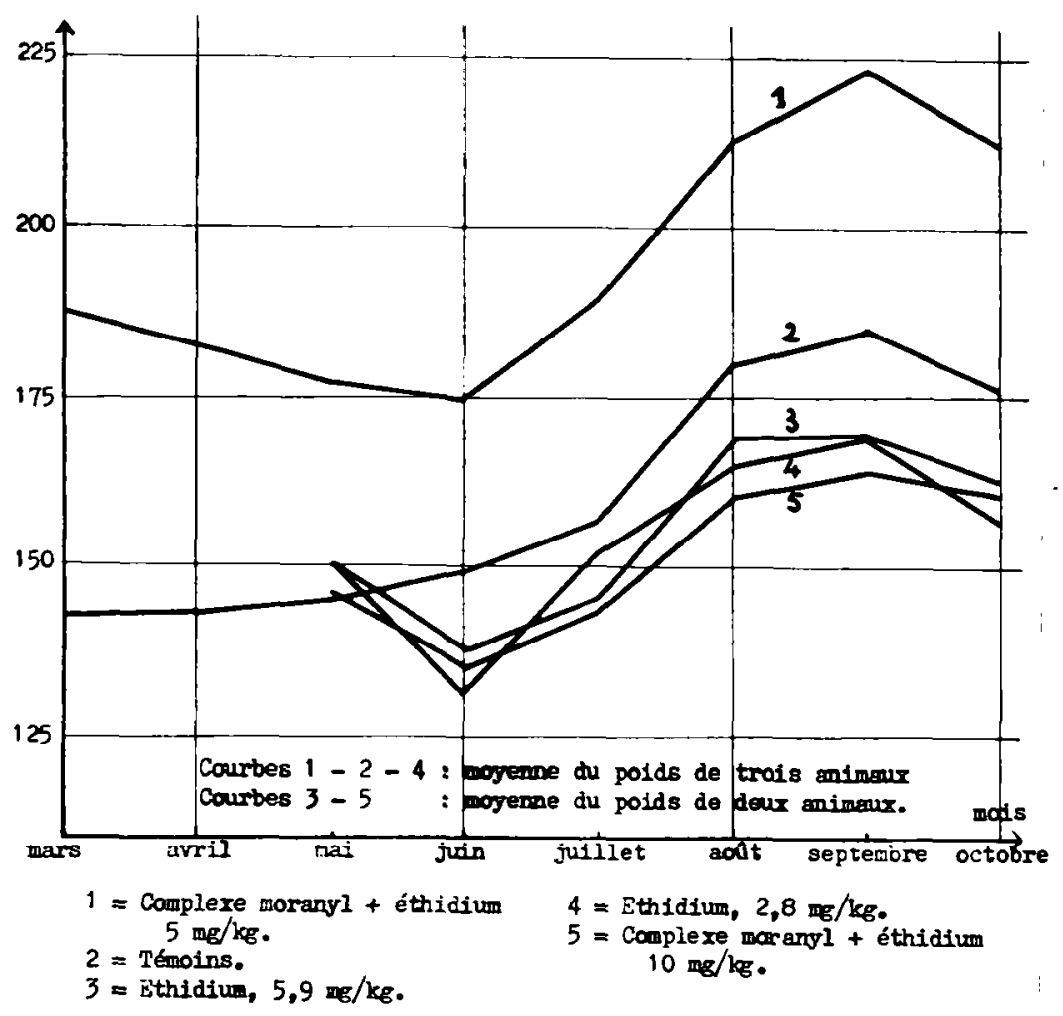




\section{CONCLLUSIONS}

a) Le moranylate d'éthidium est un corps très irritant mais qu'il est possible d'utiliser en suspension huileuse d̀ 10 p. 100 dans le fanon, à une dose ne dépassant pas $5 \mathrm{mg} / \mathrm{kg}$ pour des animaux de petite taille c'est-à-dire d'un poids de $100 \mathrm{~kg}$. Des animaux de grande taille doivent faire l'objet d'un' dosage moins important. Pratiquement, on peut adopter une dose standard de $500 \mathrm{mg}$ quel que soit l'animal, mais il estalors évident que pour un bœuf de grande taille la durée de protection sera diminuée.

b) Utilisé en suspension huileuse dans les conditions précédemment définies, il présente une toxicité générale négligeable. Cette dernière est absolument nulle pour les implants.

c) Le moranylate d'éthidium est un très bon trypanocide.

d) Comme trypanopréventif on peut en attendre de bons résultats en zone moyennement infestée, en utilisaṇt une suspension huileuse à. 10 p. 100 injectée au niveau du fanon avec une longue aiguille.

e) Les implants ne permettent pas à notre avis une diffusion suffisante pour obtenir une prévention absolve et bien que leur utilisation soit possible à grande échelle dans des régions peu infestées, on est en droit de se demander si une chimiorésistance consécutive à lẹur emploi n'est pas à craindre.

Une associațion dans le même implant de deux trypanopréventifs de structure chimique et d'action différentes permettrait probablement de retarder cette apparition et d'exercer une prophylaxie efficace.

(Le M et B 4404 qui est une combinaison d'éthidium et de bérénil serait à essayer).

$$
\begin{gathered}
\text { Institut d'élevage } \\
\text { et de médécine vétérinaire } \\
\text { des pays tropicaux : } \\
\text { Laboratoire de recherches vétérinaires } \\
\text { de Farcha, Fort-Lamy (Tchad) }
\end{gathered}
$$

\section{RÉSUMÉ}

Le moranylate d'éthidium est à la fois un excellent trypanocide et trypanopréventif. Il est très irritant localement mais peut être utilisé avec profit en suspension huileuse d̀ des doses inférieures à $5 \mathrm{mg} / \mathrm{kg}$ ou en implants, dans des zones d'infestation moyenne.

\section{SUMMARY}

\section{Research on Ethidium moranylate. III. Modifications in toxicity and preventive properties}

While ethidium moranylate is both an excellent trypanocide and trypano-prophylactic, it causes considerable irritation at the site of injection. This, however, may be minimised by lower doses, e. $9.5 \mathrm{mg} / \mathrm{kg}$, in an oily suspension or by implants. This dosage would be of value only in areas of average infestation:

\section{RESUMEN}

\section{Investigaciones sobre el moranilato de etidion}

\section{Modificationes de la toxicidad y poder preventivo}

El moranilato de etidion es a la vez un excelente preventivo de la tripanosomiases y tripanicida. El es muy irritante localmente pero puede ser utilizado útilmente en suspension oleosa a dosis inferiores a $5 \mathrm{mg} / \mathrm{kg}$ o en implantación, en zonas de infestación media. 


\section{BIBLIOGRAPHIE}

DESOWITZ (R. S.). - Suramin complexes. II. Prophylactic activity against Trypanosoma vivax in cattle. Ann, Trop. Med. Porasit., 1957. t. 51, p. 457-463.

FORD (J. M.), WILMSHURST (E. C.) et KARIB (A. A.). - Studies on ethidium bromide. The treatment of early $T$. vivax infection in cattle. Vet. Rec., 1953, t. 65, p. 589.

$\operatorname{LEACH}(T . M$.), KARIB (A. A.), FORD (E. J. M.) ef WILMSHURST (E. C.). - Studies on ethidium bromide. The prophylactic properties of the drugs. 1. comp. Path. Ther., 1955, t: 65. p. 130-142.

MILNE (A. H.) et ROBSON (J.). - The late treatment of Trypanosoma congolense infection in Zebu cattle with ethidium bromide. Vef.'.Rec., 1955, t. 67. p. 452.

ROBSON (J.). - A field trial of prophylactic drugs against trypanosomiasis in zebu cattle. Vet. Rec., 1958, t.: 70 , p. 925-927.

ROBSON (J.) el MILNE (A. H.). - A preliminary trial with three new drugs as prophylactics against Trypanosoma congolense in zebu cattle. Vet. Rec., 1957, t. 69, p. 564-566.

ROBSÓN (J.) ef HOPE CAWDERY (M. J.). Prophylaxis against trypanosomiasis in zebu cattle. A comparison of prothidium, the suraminates of ethidium and R. D. 2902, and antrycide prosalt. Vet. Rec., 1958 , t. 70 , p. 870-876.

SMITH (L. M.). - Chemoprophylaxis against bovine trypanosomiasis. I. Duration of protection from prothidium, and ethidium and R. D. suraminates, in an area of high tsetse density. J. comp. Path, 1959, t. 69, p. 105-115.

STEPHEN (L. E.) ef MACKENZIE (C. P.). - Trypanosoma vivax infection in a mare : treatment with ethidium bromide. Vef. Rec., 1958, t. 70, p. 293-294.

STEPHEN (L. E.) et WILLIAMSON (J.). - Suramin complexes. V. Ethidium complex : attempts to overcome the injection-site reaction in cattle. Ann. trop. Med. Porosit., 1958, t. 52, p. 427-442.

STEPHEN (L. E.), - Suramin complexes. IV. Ethidium bromide complex: a large scale laboratory trial of its prophylactic activity in cattle. Ann. trop. Med. Porasit., 1958, t. 52, p. $417-426$.
THIENPONT (D.) et HERIN (V.). - Le traitement de la trypanosomiase bovine à trypanosome vivax par le bromure d'éthidium. Le contrôle en brousse à Astrida (Ruanda-Urundi). Ann. Soc. belge Med. trop., 1955, †. 35, p. 439450.

UNSWORTH (K.). - Observations sur les effets curatifs et toxiques du bromure d'éthidium chez le bétail zébu infecté par T. vivax. Comité Sci. int. Recher. Trypanos. (B. P. I. T. T.) 1954, no 206, p. T56-157.

UNSWORTH (K.). - The curative effect of éthidium bromide against Trypanosoma vivax infections of zebu cattle in West Africa, with observations on the foxicity of the drugs. Ann. trop. Med. Porasit,, 1954, t. 48, p. 229236. Further observations on the curative effect of ethidium bromide against Trypanosoma vivax infections on zebu'cattle in west Africa. Ann. trop. Med. Porosit., 1954, t. 48, p. $237-241$.

WATSON (H. J. C.) et WILLIAMSON (J.).-Suramin complexes. III. Preliminary experiments on Trypanosoma simiae infections in pigs. Ann. trop. Med. Parasit., 1958, t. 52, p. 7281.

WILLIAMSON (J:) et DESOWITZ (R. S.). Prophylactic activity of suramin complexes in animal trypanosomiasis. Nature (Lond.) 1956, t. 177, p. 1074-1075.

WILLIAMSON (J.). - Suramin complexes. I. Prophylactic activity against Trypanosoma congolense in small animals. Ann. trop. Med. Parcsit., 1957, t. 51, p. 440-456.

WILSON (S. G.) et FAIRCLOUGH (R.). - A preliminary note on treatment of $T$. congolense infections with ethidium bromide in cattle in Kenya Colony. Vet. Rec., 1953, t. 65, p. 201.

WOOLFE (G.). - Trypanocidal action of phenanthridine compounds : effect of changing the quaternary groups of known irypanocides. Brit. J. Pharmocol., 1956, t. 11, p. 330333. Trypanocidal action of phenanthridine compounds: further $2: 7$-diamino phenanthridium compounds. Ibid., p. 334-338. 